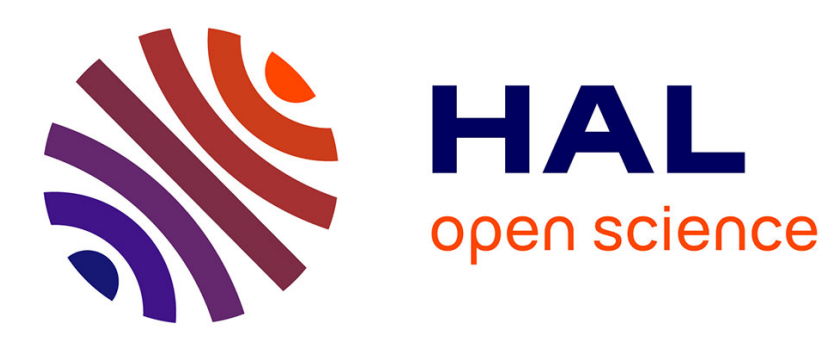

\title{
Adaptive non-separable wavelet transform via lifting and its application to Content-Based Image Retrieval
}

Gwénolé Quellec, Mathieu Lamard, Guy Cazuguel, Béatrice Cochener, Christian Roux

\section{- To cite this version:}

Gwénolé Quellec, Mathieu Lamard, Guy Cazuguel, Béatrice Cochener, Christian Roux. Adaptive nonseparable wavelet transform via lifting and its application to Content-Based Image Retrieval. IEEE Transactions on Image Processing, 2010, 19 (1), pp.25-35. 10.1109/TIP.2009.2030479 hal-00473899

\section{HAL Id: hal-00473899 \\ https://hal.science/hal-00473899}

Submitted on 16 Apr 2010

HAL is a multi-disciplinary open access archive for the deposit and dissemination of scientific research documents, whether they are published or not. The documents may come from teaching and research institutions in France or abroad, or from public or private research centers.
L'archive ouverte pluridisciplinaire HAL, est destinée au dépôt et à la diffusion de documents scientifiques de niveau recherche, publiés ou non, émanant des établissements d'enseignement et de recherche français ou étrangers, des laboratoires publics ou privés. 


\title{
Adaptive Non-Separable Wavelet Transform via Lifting and its Application to Content-Based Image Retrieval
}

\author{
Gwénolé Quellec $^{1,3}$, Mathieu Lamard ${ }^{2,3}$, Guy Cazuguel ${ }^{1,3}$, Member, IEEE, Béatrice Cochener ${ }^{2,3,4}$, \\ Christian Roux $^{1,3}$, Fellow, IEEE
}

\begin{abstract}
We present in this article a novel way to adapt a multidimensional wavelet filter bank, based on the non-separable lifting scheme framework, to any specific problem. It allows the design of filter banks with a desired number of degrees of freedom, while controlling the number of vanishing moments of the primal wavelet ( $\tilde{N}$ moments) and of the dual wavelet $(N$ moments). The prediction and update filters, in the lifting scheme based filter banks, are defined as Neville filters of order $N$ and $N$, respectively. But, in order to introduce some degrees of freedom in the design, these filters are not defined as the simplest Neville filters. The proposed method is convenient: the same algorithm is used whatever the dimensionality of the signal, and whatever the lattice used.

The method is applied to Content-Based Image Retrieval (CBIR): an image signature is derived from this new adaptive nonseparable wavelet transform. The method is evaluated on four image databases and compared to a similar CBIR system, based on an adaptive separable wavelet transform. The mean precision at five of the non-separable wavelet based system is notably higher on three out of the four databases, and comparable on the other one. The proposed method also compares favorably with the dual-tree complex wavelet transform, an overcomplete non-separable wavelet transform.
\end{abstract}

Index Terms-multidimensional wavelet, non-separable wavelet, lifting scheme, wavelet adaptation, CBIR

\section{INTRODUCTION}

Over the last decades, the wavelet transform has been widely used in many applications for its flexibility: in particular, it is possible to adapt the wavelet basis to any specific problem. However, its use has usually been restricted to 1-dimensional signals, or to separable wavelets and separable subsampling lattices in the case of multidimensional signals. Multidimensional non-separable filter banks have been studied since 1988 [1], [2] and the particular case of multidimensional nonseparable wavelets since 1992 [3], but they are not widely used yet, because of their complexity. Using separable wavelets and lattices is convenient for both designing the filters and computing the wavelet transform of signals, but it may be a limitation for some applications. In this article, we propose a generic method to adapt the wavelet transform to any specific problem, in a multidimensional non-separable setup. For this

1 INSTITUT TELECOM; TELECOM Bretagne UEB; Dpt ITI, Brest, F-29200 France;

2 Univ Bretagne Occidentale, Brest, F-29200 France; mathieu. lamardeuniv-brest. fr

3 Inserm, U650, IFR 148 ScInBioS, Brest, F-29200 France;

4 CHU Brest, Service d'Ophtalmologie, Brest, F-29200 France; purpose, we propose to implement the wavelet transform using the lifting scheme.

The lifting scheme has been proposed by Sweldens in 1996 to generate second generation wavelets [4]. The lifting scheme has many advantages over the previous approaches. In particular, all the interesting properties of wavelets, such as biorthogonality and regularity, are defined by linear relationships between the filter bank coefficients. As a consequence, it is easier to design wavelet filters. Moreover, unlike usual wavelets, checking those properties does not involve computing the Fourier transform of the wavelets. As a consequence, wavelets can be designed on arbitrary lattices. Multidimensional second generation wavelets have been adapted to non-separable lattices in 1995, on a sphere [5], [6], and in 1997, on the two-dimensional Quincunx lattice [7]. It has been generalized to any lattices in 2000 [8]. Since then, most research works on wavelets are focusing on their extensions, such as the morphological wavelets [9], the curvelets [10], the bandelets [11] or the contourlets [12]. The proposed adaptive nonseparable wavelet transform could be applied to some of these extensions.

Typically, the wavelet filter coefficients are designed in order to satisfy the biorthogonality condition and to maximize the regularity of both the primal wavelet (used for analysis) and the dual wavelet (used for synthesis) [13], [8]. However, it is possible to use some design degrees of freedom to adapt the wavelet to a particular problem. Several separable wavelet adaptation methods have been described in the literature [14], [15], in particular for second generation wavelets [13], [16]. In [14] and [15], several procedures are proposed to design wavelets, with a desired support and number of vanishing moments, that approximate a reference signal up to a desired scale. In [13], a general wavelet design framework is proposed that allows the creation of scale- or space- dependent wavelets, for instance; it has been applied to tune wavelets in order to optimize a high level criterion: the classification performance of a microaneurysm detector in eye fundus images [17]. In [16], lifting structures are proposed that makes it possible to choose between different update filters, the choice being triggered by a local gradient of the image. A second generation wavelet adaptation procedure has been proposed in 2002 for two-dimensional non-separable wavelets, on the Quincunx lattice [18], [19]. In that method, based on [8], the lifting scheme's prediction filter can be tuned by setting a multiplier between two predefined filters. A nice property of that method 
is the ability to adapt the wavelet, while preserving a desired number of vanishing moments. However, to our knowledge, no general second generation wavelet adaptation procedures have been presented for multidimensional non-separable wavelets. In this article, we propose to extend the generic method proposed by Kovacevic and Sweldens [8], in order to make it adaptive, while preserving its genericity: we don't make any assumptions on the dimension and the lattice, and the lifting scheme prediction and update filters are designed freely. So, unlike [16], [18], [19], the shape of the wavelet can be freely designed, in an automatic fashion. Unlike [14], [15], the wavelet adaptation procedure is independent of the criterion to optimize. Unlike [17], a desired number of vanishing moments can be preserved. And finally, unlike [13], [14], [15], [16], [17], the adaptation is performed for multidimensional nonseparable wavelets, and it is not restricted to the Quincunx lattice, as opposed to [18], [19].

When designing a lifting scheme filter $F$ (either a prediction or an update filter), by Kovacevic and Sweldens's method [8], all the design degrees of freedom ( $n_{F}$ degrees) are used to make the first $n_{F}$ moments of the corresponding wavelet vanish. This is done by defining $F$ as the simplest Neville filters of order $n_{F}$, with a given shift. In order to make the method adaptive, we propose to define each lifting scheme filter $F$ as a Neville filters of order $n_{F}$, but not the simplest: we use $n_{F}^{\prime}$ additional degrees of freedom to build a more complex Neville filter. Tuning these Neville filters, we can generate wavelet decompositions better suited to any specific application. Note that Neville filters, in conjunction with Lagrange interpolation, have been used to design a non-adaptive wavelet family [20], also related to the one described in [8]. In the method we propose, the design degrees of freedom that are not used to make the first wavelet moments vanish, are tuned to optimize a high-level criterion. Typically, for a compression application, this criterion would be the signal-to-noise ratio; for classification, it may be the accuracy; and for information retrieval, it may be the mean precision.

The amount of signals of various dimensions, stored in multimedia databases for many applications (medicine, defense, weather forecast, news, etc.) is increasing exponentially. In order to make the most of the available data, for data mining or information retrieval, it is necessary to develop efficient multidimensional signal processing tools. In this article, we also propose to apply our novel wavelet adaptation method to Content-Based Image Retrieval (CBIR) [21]. The goal of CBIR is to retrieve, from a database, images that are similar to an image placed as a query. Previously, we proposed a wavelet based CBIR system involving wavelet optimization [22]. The wavelet basis was searched within the one-dimensional lifting scheme framework: the wavelet functions were supposed separable; as a consequence the optimal wavelet decomposition was applied to the rows and columns of images independently. In this article, we propose to search for an adapted nonseparable wavelet basis, within the two-dimensional lifting scheme framework, for image retrieval. Note that, using the proposed wavelet adaptation method, the generalization of image retrieval in higher dimensions $(2 \mathrm{D}+\mathrm{t}, 3 \mathrm{D}, 3 \mathrm{D}+\mathrm{t}$, etc.) is straightforward. To our knowledge, multidimensional non- separable filter banks, with a varying number of degrees of freedom, have never been applied to CBIR.

The article plan is as follows. Section II presents multiresolution analysis in a multidimensional framework and section III presents the lifting scheme. Section IV summarizes how wavelets are designed in [8], and discusses the implementation choices we made. Section V explains how we propose to adapt the wavelets, while preserving a certain amount of zero moments. Some examples of multidimensional lattices are presented in section VI. The method is then applied to CBIR, in section VII, and we end up with a discussion in section VIII.

\section{Multiresolution AnAlysis}

\section{A. One-dimensional Multiresolution Analysis [17]}

A multiresolution approximation is a nested sequence of linear spaces $\left(V_{j}\right)_{j \in \mathbb{Z}}$. The approximation of a function $f \in$ $L_{2}(\mathbb{R})$ at a resolution $2^{j}$ is defined as the projection of $f$ on $V_{j} .\left(x \mapsto \Phi_{j k}(x)=\sqrt{2^{j}} \Phi\left(2^{j} x-k\right)\right)_{k \in \mathbb{Z}, \Phi \in L_{2}(\mathbb{R})}$ is a basis of $V_{j}, j \in \mathbb{Z}$, where $\Phi$, called the scaling function, satisfies the refinement relation of equation (1).

$$
\Phi(x)=2 \sum_{k=-\infty}^{\infty} h_{k} \Phi(2 x-k)
$$

The difference between two approximations of $f$, at resolutions $2^{j}$ and $2^{j+1}$, is given by the projection of $f$ on $W_{j}$, the orthogonal complement of $V_{j}$ in $V_{j+1} .\left(x \mapsto \Psi_{j k}(x)=\right.$ $\left.\sqrt{2^{j}} \Psi\left(2^{j} x-k\right)\right)_{k \in \mathbb{Z}, \Psi \in L_{2}(\mathbb{R})}$ is a basis of $W_{j}, j \in \mathbb{Z}$, where $\Psi$, called the wavelet function, satisfies the refinement relation of equation (2).

$$
\Psi(x)=2 \sum_{k=-\infty}^{\infty} g_{k} \Phi(2 x-k)
$$

\section{B. d-dimensional Multiresolution Analysis}

For a one-dimensional multiresolution analysis, the refinement relations involve downsampling by a factor of 2 , denoted $\downarrow 2$ (see equations 1,2). In higher dimensions $(d \geq 2$ ) the refinement relations involve downsampling by a dilation matrix $D \in \mathcal{M}_{d}(\mathbb{Z})$, denoted $\downarrow D$, where $\mathcal{M}_{d}(\mathbb{Z})$ denotes the set of $d$-by- $d$ matrices: $\mathbb{Z}^{d} \mapsto D \cdot \mathbb{Z}^{d}+t, t \in \mathbb{Z}^{d}$. Equations 1 and 2 are generalized as follows:

$$
\begin{aligned}
& \Phi(x)=\sum_{k=-\infty}^{\infty} h_{k} \Phi(D \cdot x-k) \\
& \Psi(x)=\sum_{k=-\infty}^{\infty} g_{k} \Phi(D \cdot x-k)
\end{aligned}
$$

Let $M$ be the absolute value of the determinant of $D$ : $M=|\operatorname{det}(D)|$. The approximation of a function $f \in L_{2}\left(\mathbb{R}^{d}\right)$ at a resolution $M^{j}$ is defined as the projection of $f$ on the basis $\left(x \mapsto \Phi_{j k}(x)=M^{\frac{j}{2}} \Phi\left(D^{j} \cdot x-k\right)\right)_{k \in \mathbb{Z}^{d}}$. The additional information available in the approximation of $f$ at the resolution $M^{j+1}$, as compared with the resolution $M^{j}$, is given by the projection of $f$ on the basis $\left(x \mapsto \Psi_{j k}(x)=\right.$ $\left.M^{\frac{j}{2}} \Psi\left(D^{j} \cdot x-k\right)\right)_{k \in \mathbb{Z}^{d}}$. 
The dilation matrix $D$ downsamples $\mathbb{Z}^{d}$ in $M$ exclusive and complementary lattices. The columns of $D\left(n_{0}, n_{1}, \ldots, n_{d-1}\right)$ are the basis vectors of the first lattice $T_{0}: T_{0}=\{D \cdot k / k \in$ $\left.\mathbb{Z}^{d}\right\}$. Lattices $T_{i}, i=1 . . M-1$, are translated from $T_{0}$ by a vector $t_{i}\left(t_{0}=0\right): T_{i}=\left\{D \cdot k+t_{i} / k \in \mathbb{Z}^{d}\right\}$. Note that different dilation matrices can lead to identical lattices.

By definition, if $D$ is diagonal, then $T_{0}, \ldots, T_{M-1}$ are separable lattices, otherwise they are non-separable lattices.

\section{Recursive Signal Analysis}

We want to compute the approximation and the details at the resolution $M^{j+1}$ of a discrete signal $f \in \mathbb{R}^{\mathbb{Z}^{d}}$ from its approximation at the resolution $M^{j}$. The coefficients of the approximation at the resolution $M^{j}$ lie in the lattice $T_{0}^{(j)}=\left\{D^{j} \cdot k / k \in \mathbb{Z}^{d}\right\}$. The coefficients of the approximation at the resolution $M^{j+1}$ lie in the lattice $T_{0}^{(j+1)}=\left\{D^{j+1}\right.$. $\left.k / k \in \mathbb{Z}^{d}\right\}$ and the coefficients of the details lie in the lattices $T_{i}^{(j+1)}=\left\{D^{j+1} \cdot k+D^{j} \cdot t_{i} / k \in \mathbb{Z}^{d}\right\}, i=1 . . M-1$. Initially $(j=0)$, the coefficients of $f$ lie in the lattice $T_{0}^{(0)}=\mathbb{Z}^{d}$.

For instance, a set of lattices is illustrated in figure 1 for $D=\left(\begin{array}{cc}2 & 2 \\ 1 & -1\end{array}\right)$ (hexagonal lattice in dimension 2): initially, the basis vectors are $\left(\left(n_{0}^{(0)}\right)\left(n_{1}^{(0)}\right)\right)=D^{0}=I_{2}$, then after the first iteration, they are $\left(\left(n_{0}^{(1)}\right)\left(n_{1}^{(1)}\right)\right)=D$, finally after the second iteration they are $\left(\left(n_{0}^{(2)}\right)\left(n_{1}^{(2)}\right)\right)=D^{2}$.

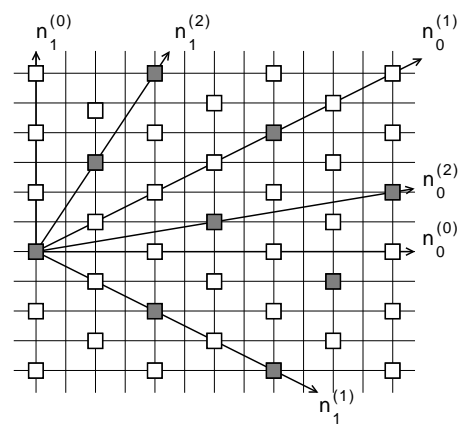

Fig. 1. Recursive signal analysis (for an hexagonal lattice in dimension 2) The lattice $T_{0}^{(0)}$ is represented by squares, the lattice $T_{0}^{(1)}$ is represented by gray squares.

\section{The LifTING SCHEME}

In 1996, Sweldens introduced a convenient way to satisfy all the desired properties of wavelets by reducing the problem to a set of simple relations between the wavelet and scaling filter coefficients, namely the lifting scheme [4]. Using the lifting scheme, it is possible to generate any compactly supported biorthogonal wavelet, with a compactly supported dual, which ensures that decomposed signals can be perfectly reconstructed. A filter bank was proposed in the one-dimensional case to implement the lifting scheme [13]. It defines two linear filters, denoted $P$ (for prediction) and $U$ (for update).

The multidimensional lifting scheme is illustrated in the case $M=2$ in figure 2 (it simply generalizes the one-dimensional case [13], replacing $\downarrow 2$ and $\uparrow 2$ by $\downarrow D$ and $\uparrow D$, respectively) and in the general case in figure 3. Filters $P_{i}$ and $U_{i}$, $i=1 . . M-1$, are prediction and update filters, respectively.

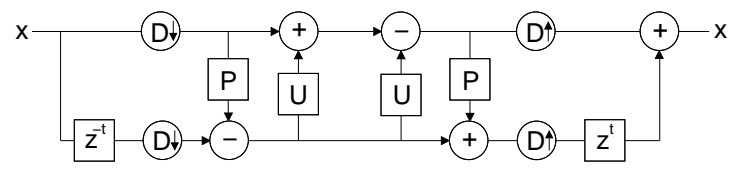

Fig. 2. 2-band lifting scheme with $t_{0}=0$ and $t_{1}=t$.

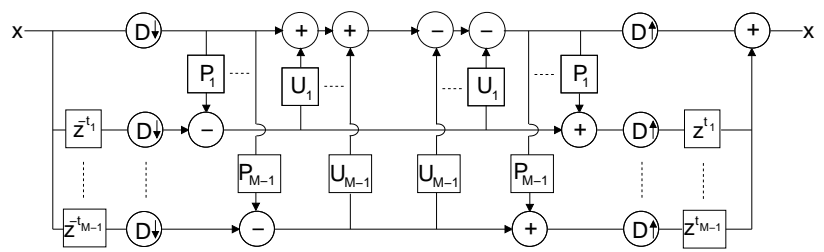

Fig. 3. $M$-band lifting scheme with $t_{0}=0$.

Definition: let $\pi(x), x \in \mathbb{R}^{d}$, be a polynomial in $x$, let $\pi\left(\mathbb{Z}^{d}\right)$ be the polynomial sequence on the lattice $\mathbb{Z}^{d}\left\{\pi(k) \mid k \in \mathbb{Z}^{d}\right\}$, and let $\Pi_{n}$ be the space of all polynomial sequences of degree strictly less than $n$. A filter $F$ is a Neville filter of order $n$ with shift $\tau \in \mathbb{R}^{d}$ if $F \pi\left(\mathbb{Z}^{d}\right)=\pi\left(\mathbb{Z}^{d}+\tau\right)$ for each polynomial sequence $\pi\left(\mathbb{Z}^{d}\right) \in \Pi_{n}$.

A wavelet has $n$ vanishing moments if and only if its scaling function can generate polynomials of degree less than or equal to $n$. This property is used to describe the approximating power of scaling functions, or equivalently, the possibility to characterize the order of isolated singularities. A sufficient condition to guarantee that the first $\tilde{N}$ moments of the primal wavelet vanish is that the prediction filters $P_{i}$ are Neville filters of order $\tilde{N}$ with shift $\tau_{i}=D^{-1} \cdot t_{i}$ [8]. And a sufficient condition to guarantee that the $N \leq \tilde{N}$ first moments of the dual wavelet vanish is that the filters $M \cdot U_{i}$ are Neville filters of order $N$ with shift $-\tau_{i}$ [8].

\section{Building A NeVille Filter}

Kovacevic and Sweldens proposed to build the prediction and update filters $P_{i}$ and $U_{i}, i=1 . . M-1$, as Neville filters [8]. A filter $F$ is a Neville filter of order $n_{F}$ with shift $\tau_{F}$ if and only if its impulse response $\left\{f_{k}\right\}$ satisfies $\sum_{k \in \mathbb{Z}^{d}} f_{-k} \prod_{i=1}^{d} k_{i}^{n_{i}}=\prod_{i=1}^{d} \tau_{F_{i}}^{n_{i}}$, for each $\sum_{i=1}^{d} n_{i}<n_{F}$, where $k_{i}$ (resp. $\tau_{F_{i}}$ ) denotes the $i^{\text {th }}$ component of $k$ (resp. $\tau_{F}$ ) and $n_{i} \in \mathbb{N}$ is the corresponding exponent [8]. Consequently, there are $C_{n_{F}-1+d}^{d}$ equations to solve.

Let $\Theta$ be a set of $n$ points. In a one-dimensional space, there is a unique polynomial of degree strictly less than $n$ to interpolate a function on $\Theta$. In a $d$-dimensional space, $d>1$, the number $n$, such that there is a unique polynomial $p$ of degree strictly less than or equal to $n\left(p \in \Pi_{n}\right)$ to interpolate a function on $\Theta$, depends on the configuration of $\Theta$. The following approach is thus applied to find a set of points $\Theta$ [8]:

- the De Boor-Ron algorithm [23] is used to compute the space $\Pi_{\Theta}$ of $\mathbb{R}^{d}$ polynomials in which there is a unique interpolant for each function defined on $\Theta$.

- the largest integer $n$ such that $\Pi_{n}$ is included in $\Pi_{\Theta}$ is looked for. The way we propose to test the inclusion of $\Pi_{n}$ in $\Pi_{\Theta}$ is presented in appendix $A$, after a brief introduction to the De Boor-Ron algorithm. 
- the neighborhood $\Theta$ is increased until the desired order $n$ is reached.

The neighborhood of $\tau_{F}$ is searched for in the span of an invertible matrix $T \in \mathcal{M}_{d}(\mathbb{Z})\left(T=I_{d}\right.$ or $T=D$, typically). In our implementation of the method, we chose the matrix $T$ (either $I_{d}$ or $D$ ) that requires the smallest neighborhood $\Theta_{F}$ of $\tau_{F}$ so that $\Pi_{n_{F}}$ is included in $\Pi_{\Theta_{F}}$, where $n_{F}$ is the desired filter order, i.e. the number of wavelet vanishing moments. In this way, the complexity of the wavelet transform algorithm is reduced.

To compute the Neville filter, the following system has to be solved:

$$
\left\{\begin{array}{l}
A=\left(\prod_{i=1}^{d} k_{i}^{n_{i}}\right)_{\sum_{i=1}^{d} n_{i}<n_{F}, k \in \Theta_{F}} \\
b=\left(\prod_{i=1}^{d} \tau_{F_{i}}^{n_{i}}\right)_{\sum_{i=1}^{d} n_{i}<n_{F}} \\
A \cdot F=b
\end{array}\right.
$$

The number of equations is $e=C_{n_{F}+d-1}^{d}$ and the number of unknowns in this system is $u=\left|\Theta_{F}\right|$, i.e. the size of the support of $F$. Since $\Pi_{n_{F}}$ is included in $\Pi_{\Theta_{F}}$, the uniqueness of the solution is ensured (whether $e$ is greater or less than $u$ ). Since $e$ and $u$ are not necessarily equal, we propose to solve this system thanks to the singular value decomposition of $A$ [24]: $A=U \cdot \operatorname{diag}\left(w_{i}\right) \cdot V^{t}, U \in \mathcal{M}_{e, u}(\mathbb{R}), V \in \mathcal{M}_{u}(\mathbb{R})$, $w \in \mathbb{R}^{u}$ ( $U$ and $V$ are orthogonal matrices). The solution of this system is given by $F=V \cdot \operatorname{diag}\left(\frac{1}{w_{i}}\right) \cdot\left(U^{t} \cdot b\right)$.

\section{Optimizing the Wavelet Filter}

We have now seen how to design wavelets with a maximum number of vanishing moments. In this section, we propose a novel solution to adapt the wavelet to any specific problem: we introduce additional design degrees of freedom to modify the Neville filter.

To adapt a filter $F$ (either $P_{i}$ or $U_{i}, i=1 . . M-1$ ) we optimize a real valued vector, whose size is the desired number $n_{F}^{\prime}$ of additional degrees of freedom for filter adaptation. Any optimization algorithm which does not require evaluating the gradient can be used, such as a genetic algorithm [25] or the Powell direction set method [26], for instance.

In practice, we do not adapt each filter independently, we adapt the whole filter bank: as a consequence, the dimension of the optimization problem is $\sum_{i=1}^{M} n_{P_{i}}^{\prime}+n_{U_{i}}^{\prime}$. So, each time the chosen optimization algorithm generates a vector, we build each filter in the filter bank with the corresponding coefficients in the generated vector, as described below, and the whole filter bank is evaluated by a problem-specific criterion.

We propose the following approach to build a filter $F$ from the corresponding coefficients in the generated vector (see figure 4):

1) we compute $\Theta_{F 1}$, the smallest neighborhood such that $\Pi_{n_{F}} \subset \Pi_{\Theta_{F 1}}$ (see section above),

2) we add new points $\Theta_{F 2}$ in the neighborhood of $\tau_{F}$ $\left(\Theta_{F}=\Theta_{F 1} \cup \Theta_{F 2}\right)$,

3) we set the filter coefficients associated with these new points $\left(F_{2}\right)$ to values generated by the optimization algorithm,
4) we modify the right-hand side of the linear system accordingly (as explained below),

5) we compute the other filter coefficients $\left(F_{1}\right)$ such that $F$ is a Neville filter of order $n_{F}$ : we solve the $e \times u$ linear system with the matrix $A$ introduced in the previous section and the new right-hand side.

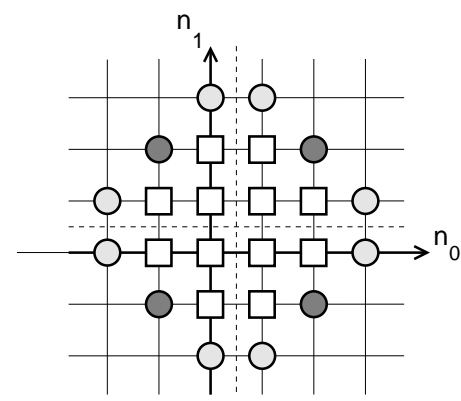

Fig. 4. Optimizing a Neville filter with shift $\tau_{F}=\left(\frac{1}{2}, \frac{1}{2}\right)$ and two degrees of freedom. The subset $\Theta_{F 1}$ of the neighborhood is represented by squares and the subset $\Theta_{F 2}$ by circles. The filter taps represented by dark gray circles (resp. light gray circles) are set to the first value $v_{1}$ (resp. the second value $\left.v_{2}\right)$ associated to this filter in the optimization vector.

As a consequence, we build a new Neville filter, with the same order $n_{F}$ and the same shift $\tau_{F}$ than the non-adapted wavelet (whose computation is described in the previous section), but this filter is no longer the simplest Neville filter of order $n_{F}$ with shift $\tau_{F}$.

$n_{F}^{\prime}$, the desired number of degrees of freedom of the filter adaptation, is the number of filter coefficient values $v_{1}, \ldots, v_{n_{F}^{\prime}}$ that we add to filter $F$. Note that $n_{F}^{\prime}$ is not the number of coefficients (several coefficients have the same value). Precisely, $v_{1}$ is associated in filter $F$ with the closest points to $\tau_{F}$, equidistant to $\tau_{F}$, outside of $\Theta_{F 1}$, we denote this set of points $P_{1} . v_{2}$ is associated in filter $F$ to the closest points to $\tau_{F}$, equidistant to $\tau_{F}$, outside of $\Theta_{F 1} \cup P_{1}$, we denote this set of points $P_{2}$, etc. $\Theta_{F 2}=P_{1} \cup P_{2} \cup \ldots \cup P_{n_{F}^{\prime}}$.

To compute the new Neville filter, we have to solve the following system, where $n_{F}$ is the order of the filter, i.e. the number of wavelet vanishing moments, and $F_{1}$ are the unknowns:

$$
\left\{\begin{array}{l}
A_{1}=\left(\prod_{i=1}^{d} k_{i}^{n_{i}}\right)_{\sum_{i=1}^{d} n_{i}<n_{F}, k \in \Theta_{F 1}} \\
A_{2}=\left(\prod_{i=1}^{d} k_{i}^{n_{i}}\right)_{\sum_{i=1}^{d} n_{i}<n_{F}, k \in \Theta_{F 2}} \\
b=\left(\prod_{i=1}^{d} \tau_{F_{i}}^{n_{i}}\right)_{\sum_{i=1}^{d} n_{i}<n_{F}} \\
A_{1} \cdot F_{1}=b-A_{2} \cdot F_{2} \\
F=\left\{F_{1} F_{2}\right\}
\end{array}\right.
$$

It also has a unique solution and we propose to solve it with the singular value decomposition method. The proposed adaptation procedure ensures that all the filter taps, equidistant to the Neville filter shift, are set to the same coefficient.

We explain in section VII-E how the proposed wavelet adaptation procedure is applied to Content-Based Image Retrieval.

\section{Some LATtices}

In the last two sections, we have seen how to design a multidimensional wavelet using the lifting scheme, and we 
have proposed a novel way to adapt it to any specific problem, regardless of the lattice used. In this section, we present some known lattices that will be compared on an application in the next section.

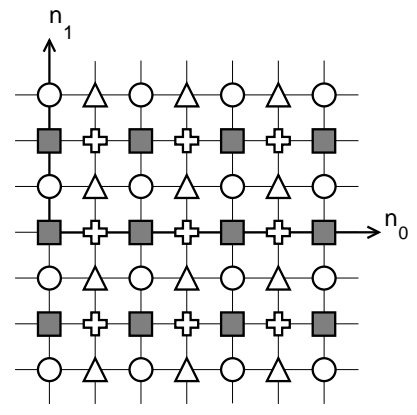

(a) separable lattice

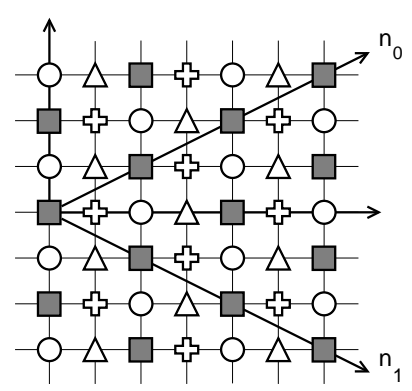

(c) hexagonal lattice

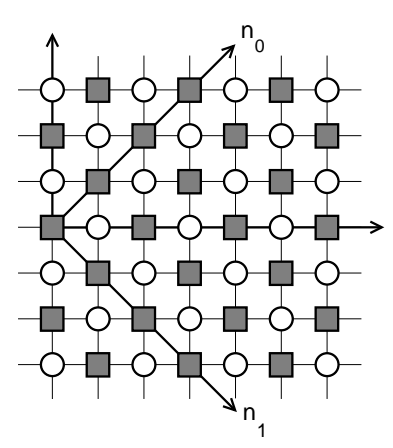

(b) Quincunx lattice

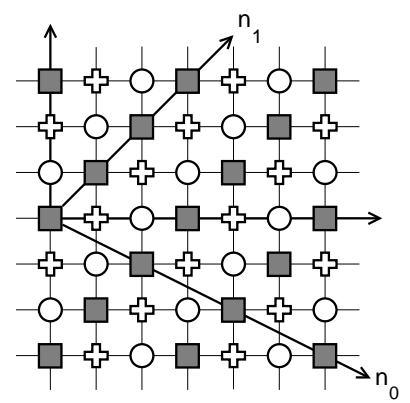

(d) triangular face lattice
Fig. 5. Examples of lattices. In each figure, the first lattice $\left(T_{0}\right)$ is represented by gray squares and each lattice is represented by distinct shapes.

\section{A. Separable Lattices}

We consider a dilation matrix $D=2 I_{d}$, where $I_{d}$ is the $d$ dimensional identity matrix. $D$ is diagonal, as a consequence the lattice is separable. The number of decomposition subbands at each scale is $M=2^{d}$. It is illustrated in the case $d=2$ below (see figure $5(\mathrm{a})$ ):

$$
D=\left(\begin{array}{cc}
2 & 0 \\
0 & 2
\end{array}\right), D^{-1}=\left(\begin{array}{cc}
\frac{1}{2} & 0 \\
0 & \frac{1}{2}
\end{array}\right)
$$

The shifts between subbands are: $t_{0}=(0,0), t_{1}=(1,0), t_{2}=$ $(0,1)$ and $t_{3}=(1,1)$; the corresponding Neville filter shifts are: $\tau_{0}=(0,0), \tau_{1}=\left(\frac{1}{2}, 0\right), \tau_{2}=\left(0, \frac{1}{2}\right)$ and $\tau_{3}=\left(\frac{1}{2}, \frac{1}{2}\right)$.

\section{B. Checkerboard Lattices}

Two expressions are possible for the dilation matrix of checkerboard lattices [3], including:

$$
D=\left(\begin{array}{ccccc}
1 & 0 & \ldots & 0 & 1 \\
1 & 1 & \ldots & 0 & 0 \\
0 & 1 & \ldots & 0 & 0 \\
\vdots & \vdots & \ddots & \vdots & \vdots \\
0 & 0 & \ldots & 1 & 0 \\
0 & 0 & \ldots & 1 & (-1)^{d-1}
\end{array}\right)
$$

Whatever the expression, $D$ is not diagonal, as a consequence it is a non-separable lattice. The number of decomposition subbands $M$ at each scale is dimension independent: $M=2$. In the case $d=2$, the checkerboard lattice is called Quincunx lattice and in the case $d=3$, it is called face centered orthorhombic lattice. It is illustrated in the case $d=2$ below (see figure 5 (b)), using equation 8 :

$$
D=\left(\begin{array}{cc}
1 & 1 \\
1 & -1
\end{array}\right), D^{-1}=\left(\begin{array}{cc}
\frac{1}{2} & \frac{1}{2} \\
\frac{1}{2} & -\frac{1}{2}
\end{array}\right)
$$

The shifts between subbands are: $t_{0}=(0,0)$ and $t_{1}=(1,0)$; the corresponding Neville filter shifts are: $\tau_{0}=(0,0)$ and $\tau_{1}=\left(\frac{1}{2}, \frac{1}{2}\right)$.

\section{Hexagonal Lattice in dimension 2}

The dilation matrix is the following [8] (see figure 5 (c)):

$$
D=\left(\begin{array}{cc}
2 & 2 \\
1 & -1
\end{array}\right), D^{-1}=\left(\begin{array}{cc}
\frac{1}{4} & \frac{1}{2} \\
\frac{1}{4} & -\frac{1}{2}
\end{array}\right)
$$

$D$ is not diagonal, as a consequence it is a non-separable lattice. The number of decomposition subbands at each scale is $M=4$. The shifts between subbands are: $t_{0}=(0,0)$, $t_{1}=(1,0), t_{2}=(2,0)$ and $t_{3}=(3,0)$; the corresponding Neville filter shifts are: $\tau_{0}=(0,0), \tau_{1}=\left(\frac{1}{4}, \frac{1}{4}\right), \tau_{2}=\left(\frac{1}{2}, \frac{1}{2}\right)$ and $\tau_{3}=\left(\frac{3}{4}, \frac{3}{4}\right)$.

\section{Triangular Face Lattice in dimension 2}

The dilation matrix is the following [8] (see figure 5 (d)):

$$
D=\left(\begin{array}{cc}
2 & 1 \\
-1 & 1
\end{array}\right), D^{-1}=\left(\begin{array}{cc}
\frac{1}{3} & -\frac{1}{3} \\
\frac{1}{3} & \frac{2}{3}
\end{array}\right)
$$

$D$ is not diagonal, as a consequence it is a non-separable lattice. The number of decomposition subbands at each scale is $M=3$. In this configuration, each point on a lattice is at the centre of a triangle in both other lattices at the same scale. The shifts between subbands are: $t_{0}=(0,0), t_{1}=(1,0)$ and $t_{2}=(2,0)$; the corresponding Neville filter shifts are: $\tau_{0}=(0,0), \tau_{1}=\left(\frac{1}{3}, \frac{1}{3}\right)$ and $\tau_{2}=\left(\frac{2}{3}, \frac{2}{3}\right)$.

\section{Application to Content-Based Image RETRIEVAL}

\section{A. Content-Based Image Retrieval (CBIR)}

CBIR is a very active research topic in all the fields where images carry relevant information [21], particularly in medicine, where imaging is present for diagnosis, therapy or education [27]. The principle of CBIR is to use images as queries to access relevant information in databases. Precisely, the goal is to retrieve similar images from these databases. The central point of CBIR is to define a similarity measure between images. In that purpose, relevant features from both the query image and images stored in the database are extracted. Typically, features characterizing shapes [28], edges in particular [29], color [30], or texture [31], are extracted. Then, the distances between feature vectors (also referred to as image signatures) are computed, and images minimizing the distance to the query are retrieved. Relevance feedback is sometimes applied, in order to enhance the semantic meaningfulness of 
retrieved images [32]. Recently, CBIR has been extended to the retrieval of multimodal documents, such as image series with metadata [33] or video with sound and text [34].

\section{B. Wavelet Based CBIR}

In this article, we apply the indexing and retrieval method proposed by Do and Vetterli, for texture retrieval [31], to image retrieval in general. To build image signatures, the principle is to model the wavelet coefficients in each subband by a generalized Gaussian distribution:

$$
\begin{gathered}
p(x ; \alpha, \beta)=\frac{\beta}{2 \alpha \Gamma\left(\frac{1}{\beta}\right)} e^{-\left(\frac{|x|}{\alpha}\right)^{\beta}} \\
\text { with } \Gamma(z)=\int_{0}^{\infty} e^{-t} t^{z-1} d t, z>0
\end{gathered}
$$

In the lower frequency subband, we model the coefficient distribution by a histogram. As a result, each image signature consists of $N-1$ maximum likelihood estimators $(\hat{\alpha}, \hat{\beta})$ of a generalized Gaussian distribution and one histogram, where $N$ is the number of decomposition subbands.

The divergence between two image signatures is defined as a weighted sum of the divergences between the coefficient distribution in the corresponding subbands of the two images. The Kullback-Leibler divergence was used to estimate the divergence between two wavelet coefficient distributions [31]. Although this divergence is asymmetric, it is not a problem in an image retrieval framework, which is asymmetric by its nature [35].

\section{Wavelet Adaptation for CBIR}

In CBIR, many factors (shape, texture, etc) come into play when we try to retrieve semantically relevant images, so it is usually complex to define a good image signature for any database. To address this problem, we can either extract features that are known to be relevant for a particular database, when expert knowledge is available, or we can design a generic image signature and tune it automatically to each database. We explored the second approach in this article. The wavelet's degrees of freedom, that we introduced in section $\mathrm{V}$, are used to tune the generic image signature, described in the previous section, to any database.

\section{The Databases}

The proposed method was applied to two medical databases (DRD and DDSM) and two non-medical databases (FD and VisTex).

1) Diabetic Retinopathy Database (DRD): this database has been built at the LaTIM laboratory (INSERM U650) for research on diabetic retinopathy follow up. Diabetic retinopathy is the main cause of blindness before 50 years old in the developed countries. The database consists of 63 files of diabetic patient examined in Brest University Hospital from June 2003 to April 2007. It contains 1,045 photographs altogether, with associated anonymous information on the pathology. Images have a definition of 1,280 pixels/rows for 1,008 rows/image. They were acquired by experts using a
Topcon Retinal Digital Camera (TRC-50IA) connected to a computer and are lossless compressed. Four types of images are obtained: color, red-free, blue-light images and angiographic sequences. An image series is given in figure 6 as an illustration. The disease severity level, ranging from 0 to 5, according to ICDRS classification [36], was determined by experts for each patient.
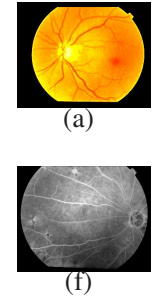

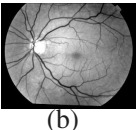

(b)

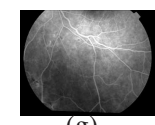

(g)

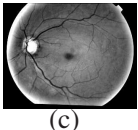

(c)

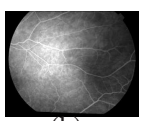

(h)

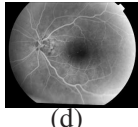

(d)

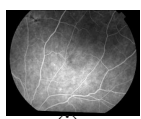

(1)

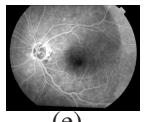

(e)

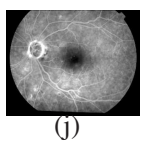

Fig. 6. Photograph series of a patient eye. Images (a), (b) and (c) are photographs obtained by applying different color filters on the camera lens. Images (d)-(j) form a temporal angiographic series: a contrast product is injected and photographs are taken at different stages (early (d), intermediate (e)-(i) and late (j)).

2) Digital Database for Screening Mammography (DDSM): the DDSM project [37], involving the Massachusetts General Hospital, the University of South Florida and the Sandia National laboratories, has built a mammographic image database for research on breast cancer screening. It consists of 2,500 patient files. Each one includes two images of each breast, along with some associated patient information. As a consequence, the database consists of 10,000 images. These images have various definitions, approximately 2,000 pixels/row for 5,000 rows/image. An example of image sequence is given in figure 7. Each patient file has been graded by a physician. Patients are then assigned one of these labels: normal, benign or cancer.

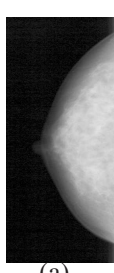

(a)

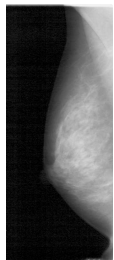

(b)

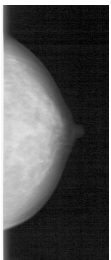

(c)

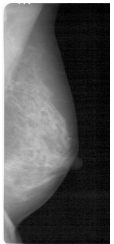

(d)
Fig. 7. Mammographic image sequence of the same patient. (a) and (b) are images of the left breast, (c) and (d) are images of the right one.

3) Face Database (FD): the database consists of 400 images: ten photographs of 40 distinct subjects ${ }^{1}$. For some of them, the images were taken at different times, with different lightings, facial expressions (open / closed eyes, smiling / not smiling) and facial details (glasses / no glasses). All the images were taken against a dark homogeneous background with the subjects in an upright, frontal position. Images have a definition of 92 pixels/row for 112 rows/image. Figure 8 shows the 10 face images of a subject. We consider that images belong to the same class if and only if they represent the same subject.

\footnotetext{
${ }^{1}$ http://www.cl.cam.ac.uk/research/dtg/attarchive/facedatabase.html
} 


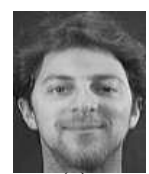

(a)

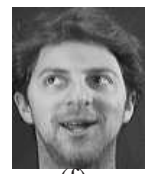

(f)

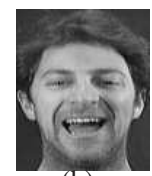

(b)

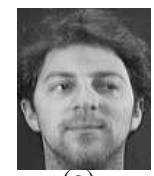

(g)

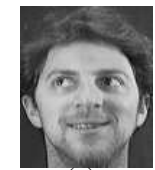

(c)

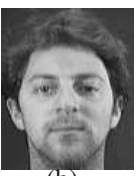

(h)

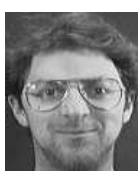

(d)

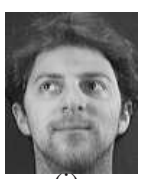

(1)

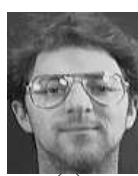

(e)

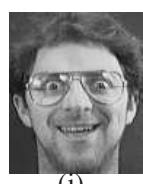

(j)
Fig. 8. Image sequence of the same person's face

4) Vision Texture database (VisTex): the database is a collection of texture images representative of real world conditions $^{2}$. Images have a definition of $512 \times 512$ pixels. We discarded the miscellaneous classes (Misc and WheresWaldo) and the classes consisting of three elements or less (Clouds, Grass and Wood), as a consequence 14 classes were selected, consisting of 153 images in total. Only the green channel of images is used in this experiment. An example is given in figure 9.

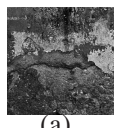

(a)

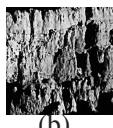

(b)

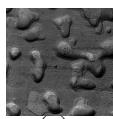

(c)

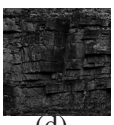

(d)

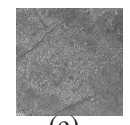

(e)

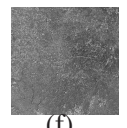

(f)
Fig. 9. Example of class from the VisTex dataset (Stone)

\section{E. Objective and tuning of the System}

In DRD, the number of cases retrieved by the system is set to five, at ophthalmologists' request: because examining retrieved images may be time consuming, they expect to find interesting images among the topmost results. For comparison purposes, the same number of cases is retrieved for the other three databases. As a consequence, the satisfaction of user's needs can be meaningfully assessed by the precision at five, the percentage of cases relevant for the query among the topmost five results.

Regarding our wavelet adaptation problem, it means that we will search for the wavelet basis that maximizes the mean precision at five of the retrieval method, in conjunction with the best set of weights between the subbands in the divergence measure (see section VII-B). The wavelet filter coefficients and the set of weights are tuned offline on a reference database, using a genetic algorithm, and this optimal setup is applied to each query image (see figure 10). As a consequence, wavelet adaptation is not performed for each image individually, but for a given dataset taken as a whole. The system is evaluated on each dataset using a 5-fold cross validation.

\section{F. Results}

We first give the performance of the system, according to the lattice used and the number $N_{l}$ of decomposition levels,

\footnotetext{
${ }^{2}$ http://vismod.media.mit.edu/vismod/imagery/VisionTexture/vistex.html
}

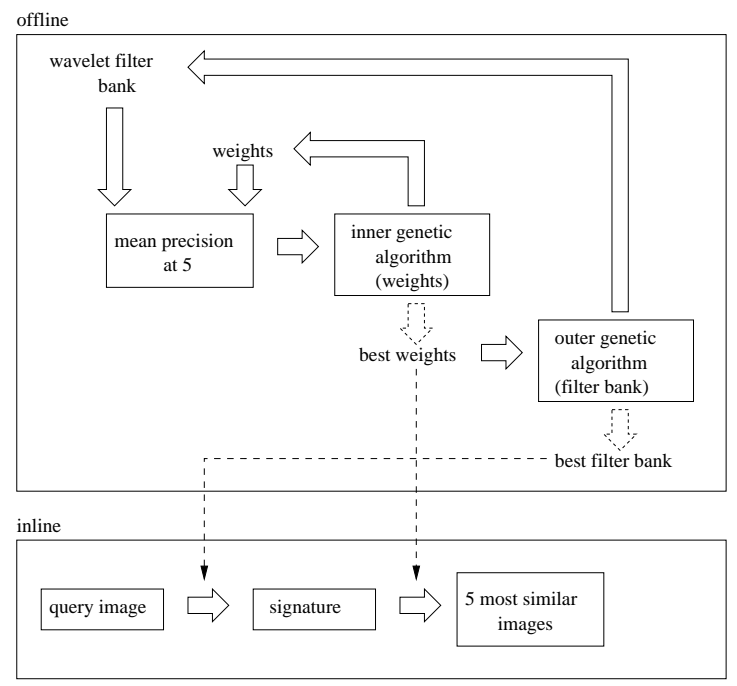

Fig. 10. Optimization Process

without filter adaptation. In order to compare the performance of the system with different lattices and numbers of decomposition levels, we propose the following definition of the scale of analysis:

$$
\text { scale }=\sqrt{M}^{N_{l}}
$$

The performance of the retrieval system according to the scale of analysis, for each lattice, is given on figure 11. The order of the prediction and update filters is set to 4 .

As the number of decomposition levels increases, the dimension of the search space increases, and it becomes harder to find good weight vectors between subbands. So, we usually observe a decrease in the mean precision at some point. For FD, the small definition of images also contributes to the decrease of the mean precision, since the low frequency subband, whose definition decreases with the number of decomposition levels, is particularly relevant for this database.

Then, for each lattice, we adapt the filter bank with the best number of decomposition levels, as obtained on figure 11. For this purpose, we add one degree of freedom per filter in the filter bank. The retrieval performance of the adapted system is given in table $\mathrm{I}$.

TABLE I

RETRIEVAL PERFORMANCE OF THE SYSTEM WITH FILTER ADAPTATION

\begin{tabular}{|r|c|c|c|c|c|}
\hline lattice & adaptation & DRD & DDSM & FD & VisTex \\
\hline \hline \multirow{2}{*}{ separable } & no & $47.74 \%$ & $71.74 \%$ & $94.85 \%$ & $57.11 \%$ \\
\cline { 2 - 6 } & yes & $48.25 \%$ & $76.03 \%$ & $95.20 \%$ & $59.08 \%$ \\
\hline \hline \multirow{2}{*}{ Quincunx } & no & $48.08 \%$ & $73.06 \%$ & $94.90 \%$ & $56.71 \%$ \\
\cline { 2 - 6 } & yes & $48.42 \%$ & $76.20 \%$ & $95.25 \%$ & $59.08 \%$ \\
\hline \hline \multirow{2}{*}{ hexagonal } & no & $46.84 \%$ & $70.91 \%$ & $95.35 \%$ & $57.24 \%$ \\
\cline { 2 - 6 } & yes & $48.47 \%$ & $71.07 \%$ & $95.35 \%$ & $58.68 \%$ \\
\hline \hline \multirow{2}{*}{ triangular } & no & $48.02 \%$ & $70.41 \%$ & $95.15 \%$ & $55.66 \%$ \\
\cline { 2 - 6 } & yes & $48.64 \%$ & $77.69 \%$ & $95.35 \%$ & $56.03 \%$ \\
\hline
\end{tabular}

We illustrate in table II the influence of the number of degrees of freedom on the mean precision score. The experiment is carried out on the face database. For each lattice, the filter bank is adapted with $p$ (resp. $u$ ) degrees of freedom 


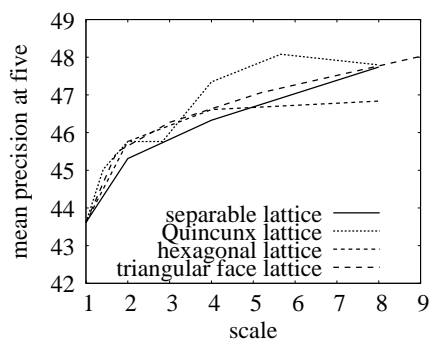

(a) DRD

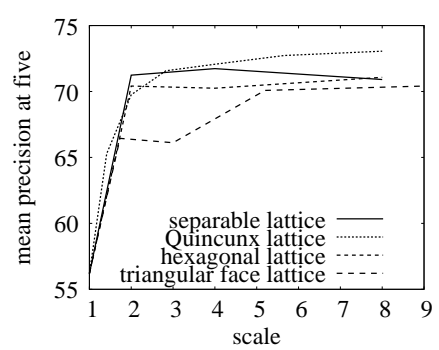

(b) DDSM

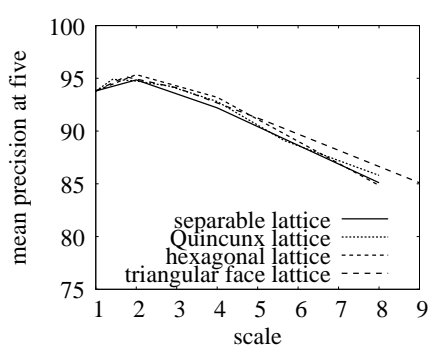

(c) $\mathrm{FD}$

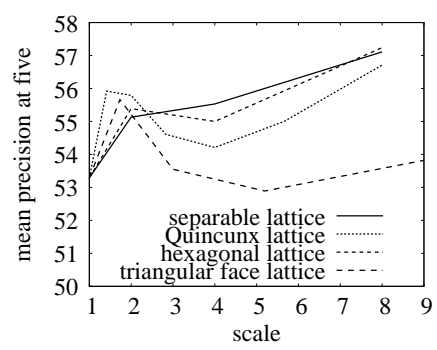

(d) VisTex

Fig. 11. Retrieval performance of the system without filter adaptation.

for each prediction (resp. update) filter, using the best number of decomposition levels. This table shows that increasing the number of degrees of freedom, up to a certain number, leads to an improvement of the performance. Anyway, if we add too many degrees of freedom, the objective function becomes more complex and it is harder to find the optimum: the system performance stops increasing and even decreases. One or two degrees of freedom for both the prediction and the update filter seems to be a reasonable choice.

TABLE II

RETRIEVAL PERFORMANCE OF THE SYSTEM ACCORDING TO THE NUMBER OF DEGREES OF FREEDOM

\begin{tabular}{|r|c||c|c|c|c|}
\hline lattice & $p \backslash u$ & 0 & 1 & 2 & 3 \\
\hline \hline \multirow{3}{*}{ separable } & 0 & $94.85 \%$ & $95.15 \%$ & $94.80 \%$ & $94.65 \%$ \\
\cline { 2 - 6 } & 1 & $95.00 \%$ & $\mathbf{9 5 . 2 0 \%}$ & $94.85 \%$ & $94.80 \%$ \\
\cline { 2 - 6 } & 2 & $\mathbf{9 5 . 2 0 \%}$ & $95.10 \%$ & $94.25 \%$ & $\mathbf{9 5 . 2 0 \%}$ \\
\cline { 2 - 6 } & 3 & $95.00 \%$ & $94.85 \%$ & $90.85 \%$ & $94.50 \%$ \\
\hline \hline \multirow{5}{*}{ Quincunx } & 0 & $94.90 \%$ & $95.20 \%$ & $95.20 \%$ & $95.05 \%$ \\
\cline { 2 - 6 } & 1 & $95.20 \%$ & $95.25 \%$ & $95.15 \%$ & $95.25 \%$ \\
\cline { 2 - 6 } & 2 & $95.30 \%$ & $95.30 \%$ & $95.20 \%$ & $95.05 \%$ \\
\cline { 2 - 6 } & 3 & $95.35 \%$ & $95.45 \%$ & $95.20 \%$ & $\mathbf{9 5 . 5 5 \%}$ \\
\hline \hline \multirow{3}{*}{ triangular face } & 0 & $95.35 \%$ & $95.65 \%$ & $94.95 \%$ & $95.2 \%$ \\
\cline { 2 - 6 } & 1 & $95.75 \%$ & $95.35 \%$ & $95.45 \%$ & $95.45 \%$ \\
\cline { 2 - 6 } & 2 & $94.90 \%$ & $95.40 \%$ & $95.25 \%$ & $95.20 \%$ \\
\cline { 2 - 6 } & 3 & $\mathbf{9 5 . 8 0 \%}$ & $95.60 \%$ & $95.30 \%$ & $94.50 \%$ \\
\cline { 2 - 6 } & 0 & $95.15 \%$ & $95.35 \%$ & $95.15 \%$ & $95.35 \%$ \\
\cline { 2 - 6 } & 2 & $95.30 \%$ & $95.35 \%$ & $94.50 \%$ & $95.25 \%$ \\
\hline \hline
\end{tabular}

The retrieval performance of the system is compared in table III to that of the separable wavelet transform based system [22] (a one-dimensional wavelet transform is applied to the rows and to the columns of the image, separately). In both cases, an adapted wavelet transform is searched for within the lifting scheme framework, and a set of weights between subbands is tuned, using exactly the same procedure (see figure 10).

TABLE III

NON-SEPARABLE VERSUS SEPARABLE WAVELET TRANSFORM - MEAN PRECISION AT 5

\begin{tabular}{|r||c|c|c|c|}
\hline wavelet transform & DRD & DDSM & FD & VisTex \\
\hline \hline separable & $46.10 \%$ & $70.91 \%$ & $95.50 \%$ & $56.05 \%$ \\
\hline non-separable & $48.64 \%$ & $77.69 \%$ & $95.35 \%$ & $59.08 \%$ \\
\hline
\end{tabular}

Using a non-separable wavelet transform, we observe a noticeable improvement of the performance on three out of the four databases (DRD, DDSM and VisTex) and the results are similar on the other one. The improvement is particularly important for DDSM; our explanation is that the tissues, the cancerous tissues in particular, tend to be aligned in an oblique direction (see figure 12 - the cancerous tissues are overlaid). As a consequence, a separable wavelet is not particularly suited. For FD, the $\mathrm{x}$-axis and the $\mathrm{y}$-axis are probably dominant, so a separable wavelet is better suited than for DDSM. And for DRD and VisTex, there are no obvious dominant directions on the dataset taken as a whole.

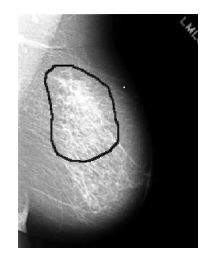

Fig. 12. Dominant direction in DDSM

The mean time required to decompose an image using the adapted separable or non-separable wavelet transform is given in table IV. Note that the time required to decompose images does not differ whether or not an adapted wavelet is used, since the wavelet is adapted to a reference database, offline, and not to each individual query image (see section VII-E). All experiments were conducted using one core of an Intel Core 2 duo E4500 based computer running at $2.2 \mathrm{GHz}$. Note that our implementation of the separable wavelet transform is based on the computationally efficient JPEG-2000 standard and that our implementation of the non-separable wavelet transform was designed to manage any dimensions and lattices; it could be optimized for a specific lattice.

TABLE IV

NON-SEPARABLE VERSUS SEPARABLE WAVELET TRANSFORM COMPUTATION TIMES

\begin{tabular}{|r||c|c|c|c|}
\hline wavelet transform & DRD & DDSM & FD & VisTex \\
\hline \hline separable & $0.32 \mathrm{~s}$ & $2.92 \mathrm{~s}$ & $0.008 \mathrm{~s}$ & $0.080 \mathrm{~s}$ \\
\hline non-separable & $6.17 \mathrm{~s}$ & $60.88 \mathrm{~s}$ & $0.044 \mathrm{~s}$ & $1.172 \mathrm{~s}$ \\
\hline
\end{tabular}

Finally, the retrieval performance of the proposed method is compared to the same CBIR framework, but based on an overcomplete wavelet transform, the 2-D dual-tree complex wavelet transform [38], since overcomplete wavelet transforms are renowned for being particularly suited for CBIR. The experiment was carried out on the VisTex dataset, with the 
Polytechnic Institute of NYU's implementation of the 2-D dual-tree complex wavelet transform ${ }^{3}$, using the Farras nearly symmetric filters [39]. The best retrieval performance was obtained for two levels of decomposition. The retrieval results are compared in table V. An improvement is observed when wavelet adaptation is performed.

TABLE V

COMPARISON WITH THE DUAL-TREE COMPLEX WAVELET TRANSFORM ON THE VISTEX DATASET

\begin{tabular}{|r||c|}
\hline wavelet transform & mean precision at 5 \\
\hline \hline dual-tree CWT [38] & $56.60 \%$ \\
\hline non-separable lifting-scheme [8] & $57.24 \%$ \\
\hline proposed adaptive wavelet transform & $59.08 \%$ \\
\hline
\end{tabular}

\section{DISCUSSION}

We presented in this article a novel method to adapt a multidimensional wavelet filter bank to any specific problem. The proposed method is based on the non-separable lifting scheme framework. It allows the design of filter banks with a desired number of degrees of freedom, while controlling the regularity of the primal and dual wavelets. The regularity of the wavelets is controlled by setting the number of low order moments that should vanish. The support of the wavelet functions is closely related to the sum of the number of degrees of freedom for adaptation and of the number of vanishing moments. The complexity of the system calibration (related to the number of degrees of freedom) and of the wavelet transform (related to the filters' support) can thus be controlled independently. The proposed system is convenient from an implementation point of view: the same algorithm is used whatever the dimensionality of the signal, and whatever the lattice used.

The proposed method is applied to Content-Based Image Retrieval (CBIR): an image signature is derived from an adapted non-separable wavelet transform, using four different lattices. The performances of the adapted wavelet filter bank over the non-adapted wavelet filter bank are higher for every database. The system is compared to a similar CBIR system, but using an adapted separable wavelet transform. The performances of the non-separable wavelet based system are notably higher on three out of the four databases (DDSM, DRD and VisTex), and comparable on the other one (FD). A large improvement is observed for DDSM, where there is a dominant oblique direction. We have also shown that the proposed system is better suited than the 2-D dual-tree complex wavelet transform on the VisTex dataset, using a Kullback-Leibler divergence based CBIR system.

In future studies on CBIR, we intend to apply the proposed wavelet adaptation scheme to signals of higher dimensions, such as CT scans, MRI, temporal MRI, etc. Also, the ability to adapt the wavelet, while maintaining a desired amount of zero moments, makes our framework potentially interesting for combined compression and retrieval; this potential shall be explored in future works.

\footnotetext{
${ }^{3} \mathrm{http}: / /$ taco.poly.edu/WaveletSoftware/dt2D.html
}

\section{REFERENCES}

[1] R. Ansari, H. Gaggioni, and D. le Gall, "HDTV coding using a non-rectangular subband decomposition," in Proc. SPIE Conf. Visual Communications and Image Processing, Cambridge, MA, November 1988, pp. 821-824.

[2] J. Kovacevic, M. Vetterli, and G. Karlsson, "Design of multidimensional filter banks for non-separable subsampling," in Proc. IEEE Int. Symp. on Circuits and Systems, New Orleans, LA, May 1990, pp. 2004-2008.

[3] J. Kovacevic and M. Vetterli, "Nonseparable multidimensional perfect reconstruction filter banks and wavelet bases for Rn," IEEE Trans. Inf. Theory, vol. 38, no. 2, pp. 533-555, March 1992.

[4] W. Sweldens, "The lifting scheme: a custom-design construction of biorthogonal wavelets," Appl. Comput. Harmon. Anal., vol. 3, no. 2, pp. 186-200, 1996.

[5] P. Schröder and W. Sweldens, "Spherical wavelets: efficiently representing functions on the sphere," in Computer Graphics Proceedings (SIGGRAPH 95), 1995, pp. 161-172.

[6] _ _ "Spherical wavelets: texture processing," in Rendering Techniques '95 (P. Hanrahan and W. Purgathofer, eds.), Springer Verlag, Wien, New York, August 1995.

[7] G. Uytterhoeven and A. Bultheel, "The red-black wavelet transform," Tech. Rep. 271, 1997.

[8] J. Kovacevic and W. Sweldens, "Wavelet families of increasing order in arbitrary dimensions," IEEE Trans. Image Proc., vol. 9, no. 3, pp. 480-496, March 2000.

[9] H. J. A. M. Heijmans and J. Goutsias, "Nonlinear multiresolution signal decomposition schemes-part II: morphological wavelets," IEEE Trans. Image Process., vol. 9, no. 11, pp. 1897-1913, November 2000.

[10] E. J. Candès and D. L. Donoho, "Curvelets - a surprisingly effective nonadaptive representation for objects with edges," in Curve and Surface fitting, A. Cohen, C. Rabut, and L. L. Schumaker, Eds. Vanderbilt University Press, 2000, pp. 105-120.

[11] E. le Pennec and S. Mallat, "Image compression with geometrical wavelets," in Proc. of the IEEE Int. Conf. on Image Processing (ICIP), Vancouver, vol. 1, September 2000, pp. 661-664.

[12] M. N. Do and M. Vetterli, "Contourlets: a directional multiresolution image representation," in Proc. of the IEEE Int. Conf. on Image Processing (ICIP), Rochester, vol. 1, September 2002, pp. I-357-I-360.

[13] R. Claypoole, R. Baraniuk, and R. Nowak, "Adaptive wavelet transforms via lifting," in Proc. of the IEEE Int. Conf. on Acoustics, Speech and Signal Processing, vol. 3, May 1998, pp. 1513-1516.

[14] A. H. Tewfik, D. Sinha, and P. Jorgensen, "On the optimal choice of a wavelet for signal representation," IEEE Trans. Inform. Theory, vol. 38, no. 2, pp. 747-765, March 1992.

[15] A. Gupta, S. D. Joshi, and S. Prasad, "A new method of estimating wavelet with desired features from a given signal," Signal Process., vol. 85, no. 1, pp. 147-161, January 2005.

[16] G. Piella, G. Pau, and B. Pesquet-Popescu, "Adaptive lifting schemes combining seminorms for lossless image compression," in IEEE Int. Conf. on Image Processing, ICIP 2005, vol. 1, 2005, pp. I - 753-6.

[17] G. Quellec, M. Lamard, P. M. Josselin, G. Cazuguel, B. Cochener, and C. Roux, "Optimal wavelet transform for the detection of microaneurysms in retina photographs," IEEE Trans. Med. Imaging, vol. 27, no. 9, pp. 1230-1241, September 2008.

[18] D. Seršić and M. Vrankić, "Adaptation of a 2-D nonseparable wavelet filter bank with variable number of zero moments," in Proc. of Visualization, Imaging, and Image Processing, September 2002.

[19] _ _ "Adaptation in the Quincunx wavelet filter bank with applications in image denoising," in Proc. of the 2004 Int. TICSP Workshop on Spectral Methods and Multirate Signal Processing, SMMSP 2004, 2004, pp. 245-252.

[20] D. Chen and T. Zhang, "Neville-Lagrange wavelet family for lossless image compression," Signal Process., vol. 88, no. 11, pp. 2833-2842, November 2008.

[21] A. Smeulders, M. Worring, S. Santini, A. Gupta, and R. Jain, "Contentbased image retrieval at the end of the early years," IEEE Trans. Pattern Anal. Mach. Intell., vol. 22, no. 12, pp. 1349-1380, December 2000.

[22] M. Lamard, G. Cazuguel, G. Quellec, L. Bekri, C. Roux, and B. Cochener, "Content based image retrieval based on wavelet transform coefficients distribution," in Proc. of the 29th annual Int. Conf. of the IEEE Engineering in Medecine and Biology Society, August 2007.

[23] C. de Boor and A. Ron, "Computational aspects of polynomial interpolation in several variables," Math. Comput., vol. 58, no. 198, pp. 705-727, April 1992. 
[24] G. H. Golub and W. Kahan, "Calculating the singular values and pseudoinverse of a matrix," J. of the Soc. for Ind. and Appl. Math.: Series B, Numerical Analysis, vol. 2, no. 2, pp. 205-224, January 1965.

[25] D. E. Goldberg, Genetic Algorithms in Search, Optimization and Machine Learning. Kluwer Academic Publishers, Boston, MA, 1989.

[26] W. H. Press, S. Teukolsky, W. Vetterling, and B. Flannery, Numerical Recipes in $C$ : the Art of Scientific Computing. Cambridge University Press, 1992, chapter 10.

[27] H. Müller, N. Michoux, D. Bandon, and A. Geissbuhler, "A review of content-based image retrieval systems in medical applications - clinical benefits and future directions," Int. J. Med. Informatics, vol. 73, no. 1, pp. 1-23, February 2004.

[28] S. Antani, D. J. Lee, L. R. Long, and G. R. Thoma, "Evaluation of shape similarity measurement methods for spine X-ray images," J. Vis. Commun. Image R., vol. 15, no. 3, pp. 285-302, September 2004.

[29] M. Banerjee and M. K. Kundu, "Edge based features for content based image retrieval," Pattern Recognit., vol. 36, no. 11, pp. 2649-2661, November 2003.

[30] P. T. Yap and R. Paramesran, "Content-based image retrieval using Legendre chromaticity distribution moments," in IEE Proc.: Vis. Image Signal Process., vol. 153, February 2006, pp. 17-24.

[31] M. N. Do and M. Vetterli, "Wavelet-based texture retrieval using generalized Gaussian density and Kullback-Leibler distance," IEEE Trans. Image Process., vol. 11, no. 2, pp. 146-158, February 2002.

[32] M. M. Rahman, P. Bhattacharya, and B. C. Desai, "A framework for medical image retrieval using machine learning and statistical similarity matching techniques with relevance feedback," IEEE Trans. Inf. Technol. Biomed., vol. 11, no. 1, pp. 58-69, January 2007.

[33] G. Quellec, M. Lamard, G. Cazuguel, B. Cochener, and C. Roux "Multimodal information retrieval based on DSmT. Application to computer-aided medical diagnosis," in Advances and Applications of DSmT for Information Fusion III, F. Smarandache and J. Dezert, Eds. American Research Press, 2009, ch. 18, pp. 471-502.

[34] E. Bruno, N. Moenne-Loccoz, and S. Marchand-Maillet, "Design of multimodal dissimilarity spaces for retrieval of video documents," IEEE Trans. Pattern Anal. Mach. Intell., vol. 30, pp. 1520-1533, September 2008.

[35] G. Quellec, "Indexation et fusion multimodale pour la recherche d'information par le contenu. application aux bases de données d'images médicales." Ph.D. dissertation, TELECOM Bretagne, Brest, France, September 2008. [Online]. Available: http://tel.archivesouvertes.fr/tel-00326131/en/

[36] C. Wilkinson, F. Ferris, R. E. Klein, P. P. Lee, C. D. Agardh, M. Davis, D. Dills, A. Kampik, R. Pararajasegaram, and J. T. Verdaguer, "Proposed international clinical diabetic retinopathy and diabetic macular edema disease severity scales," Ophthalmology, vol. 110, no. 9, pp. 1677-1682, September 2003.

[37] M. Heath, K. W. Bowyer, D. Kopans, W. P. Kegelmeyer, R. Moore, K. Chang, and S. MunishKumaran, "Current status of the Digital Database for Screening Mammography," Digital Mammography, Kluwer Academic Publishers, pp. 457-460, 1998.

[38] I. W. Selesnick, R. G. Baraniuk, and N. G. Kingsbury, "The dual-tree complex wavelet transform," IEEE Signal Process. Mag., vol. 22, no. 6, pp. 123-151, November 2005.

[39] A. F. Abdelnour and I. W. Selesnick, "Nearly symmetric orthogonal wavelet bases," in Proc. of the IEEE Int. Conf. on Acoustics, Speech, Signal Processing (ICASSP), May 2001.

\section{APPENDIX A}

\section{FINDING A BASIS TO INTERPOLATE POLYNOMIALS OF ORDER STRICTLY LESS THAN $n$}

\section{A. De Boor and Ron Algorithm}

De Boor-Ron algorithm [23] allows to build a space $\Pi_{\Theta}$ of $\mathbb{R}^{d}$ polynomials in which there is a unique interpolant for each function defined on a set $\Theta$ of points in $\mathbb{R}^{d}$. To define the space $\Pi_{\Theta}$, the following definitions are used:

- $e_{\theta_{i}}(x)=e^{\theta_{i} \cdot x}=1+\theta_{i} \cdot x+\left(\theta_{i} \cdot x\right)^{2} / 2+\ldots, x \in \mathcal{R}^{d}$, $\theta_{i} \in \Theta, \theta_{i} \cdot x=\sum_{u=1}^{d} \theta_{i}(u) x(u)$

- let $f$ be an analytic function at the origin (such as $e_{\theta_{i}}$ or a polynomial), $f \downarrow$ is the first non-zero term in the series $f=f^{(0)}+f^{(1)}+\ldots$ where $f^{(j)}$ is the sum of all the terms of degree $j$ in the power series expansion of $f$.

- $\exp _{\Theta}=\operatorname{vect}\left\{e_{\theta_{i}} \mid \theta_{i} \in \Theta\right\}$.

De Boor and Ron proved that $\Pi_{\Theta}=\left(\exp _{\Theta}\right) \downarrow$. In order to define a basis for $\Pi_{\Theta}$, they first look for a basis $\left(g_{i}\right)_{i=1 . .|\Theta|}$ for $\exp _{\Theta}$. The basis vectors $g_{i}$ are searched applying a Gaussian elimination with partial pivoting to the matrix $V$ obtained from matrix $V^{\prime}=\left(\prod_{u=1}^{d} \theta_{i}(u)^{\alpha_{j}(u)}\right)_{\theta_{i} \in \Theta, \alpha_{j} \in \mathcal{Z}^{d}}$ treating all the elements of degree $k$ as a single element: $V=\left(\prod_{u=1}^{d} \theta_{i}(u)^{\alpha_{j}(u)}\right)_{\theta_{i} \in \Theta, \sum_{u=1}^{d}} \alpha_{j}(u)=k$ (each element in $V$ is actually a vector). We obtain the factorization $V=A \cdot W$ where $A$ is an invertible scalar matrix and $W$ is a matrix of vectors in reduced row-echelon form. The basis vectors $g_{i}$ are defined as $g_{i}=x \mapsto \sum_{\alpha_{j}}\left(\prod_{u=1}^{d} x(u)^{\alpha_{j}(u)}\right) W\left(\theta_{i}, \alpha_{j}\right) / \alpha_{j} !$, $\alpha_{j} !=\prod_{u=1}^{d} \alpha_{j}(u) !$

Finally, $\Pi_{\Theta}$ is defined as $\Pi_{\Theta}=\operatorname{vect}\left\{g_{i} \downarrow|i=1 ..| \Theta \mid\right\}$.

\section{B. Testing the Inclusion of $\Pi_{n}$ in $\Pi_{\Theta}$}

Kovacevic and Sweldens [8] do not mention how they test the inclusion of $\Pi_{n}=\operatorname{vect}\left\{x \mapsto \prod_{u=1}^{d} x(u)^{\alpha(u)} \mid x \in\right.$ $\left.\mathbb{R}^{d}, \alpha \in \mathbb{Z}_{+}^{d}, \sum_{u=1}^{d} \alpha(u)<n\right\}$ in $\Pi_{\Theta}$. We propose the following approach. Let $\pi_{n^{\prime}}=\operatorname{vect}\left\{x \mapsto \prod_{u=1}^{d} x(u)^{\alpha(u)} \mid x \in\right.$ $\left.\mathbb{R}^{d}, \alpha \in \mathbb{Z}_{+}^{d}, \sum_{u=1}^{d} \alpha(u)=n^{\prime}\right\}$ be the set of all polynomials of degree $n^{\prime}$. $\Pi_{\Theta}$ is defined as the space spanned by the $g_{i} \downarrow$ defined above: $\Pi_{\Theta}=\operatorname{vect}\left\{g_{i} \downarrow|i=1 ..| \Theta \mid\right\}$. Each vector $g_{i} \downarrow$ is a linear combination of basis polynomials $\left(x \mapsto \prod_{u=1}^{d} x(u)^{\alpha(u)}\right)_{x \in \mathbb{R}^{d}, \alpha \in \mathbb{Z}_{+}^{d}, \sum_{u=1}^{d} \alpha(u)=n_{g_{i} \downarrow}}$ of $\pi_{n_{g_{i}}}$; the degree $n_{g_{i} \downarrow}$ may vary from one $g_{i} \downarrow$ to another. To test if $\Pi_{n}$ is included in $\Pi_{\Theta}$, we need to test if $\pi_{n^{\prime}} \subset \operatorname{vect}\left\{g_{i} \downarrow \mid n_{g_{i} \downarrow}=n^{\prime}\right\}$ $\forall n^{\prime} \in\{0, \ldots, n-1\}$. The space spanned by the polynomials $g_{i} \downarrow$ of degree $n^{\prime}$ is included in $\pi_{n^{\prime}}$, since each of these polynomials are defined as a linear combination of basis polynomials $\left(x \mapsto \prod_{u=1}^{d} x(u)^{\alpha(u)}\right)_{x \in \mathbb{R}^{d}, \alpha \in \mathbb{Z}_{+}^{d}, \sum_{u=1}^{d} \alpha(u)=n^{\prime}}$ of $\pi_{n^{\prime}}$. As a consequence, testing the inclusion $\pi_{n^{\prime}} \subset \operatorname{vect}\left\{g_{i} \downarrow \mid n_{g_{i} \downarrow}=n^{\prime}\right\}$ is equivalent to testing the identity $\pi_{n^{\prime}}=\operatorname{vect}\left\{g_{i} \downarrow \mid n_{g_{i} \downarrow}=\right.$ $\left.n^{\prime}\right\}$, and therefore testing if these two spaces have the same dimension.

Let $x=\left(x_{1}, x_{2}, \ldots, x_{N}\right)^{t}$ be the vector consisting of the basis polynomials of $\pi_{n^{\prime}}$ (the dimension of $\pi_{n^{\prime}}$ is $N$ ). Let $g=\left(g_{1} \downarrow, g_{2} \downarrow, \ldots, g_{M} \downarrow\right)^{t}$ be the vector consisting of the $g_{i} \downarrow$ such that $n_{g_{i} \downarrow}=n^{\prime}$. We write $g$ as the product of a matrix $A^{n^{\prime}}=\left(a_{i j}^{n^{\prime}}\right)_{i=1 . . M, j=1 . . N}$ and of $x: a_{i j}^{n^{\prime}}=\frac{W\left(\theta_{i}, \alpha_{j}\right)}{\alpha_{j} !}$ (see section $\mathrm{A}-\mathrm{A}$ ). If the rank of $A^{n^{\prime}}$ is equal to $N$, then the dimension of vect $\left\{g_{i} \downarrow \mid n_{g_{i} \downarrow}=n^{\prime}\right\}$ is $N$, and then $\pi_{n^{\prime}} \subset \operatorname{vect}\left\{g_{i} \downarrow \mid n_{g_{i} \downarrow}=n^{\prime}\right\}$.

As a conclusion, $\Pi_{n} \subset \Pi_{\Theta}$ if $\forall n^{\prime} \in\{0, \ldots, n-1\}$, the rank of $A^{n^{\prime}}$ is equal to the dimension of $\pi_{n^{\prime}}$.

To compute the rank of a matrix $A(M \times N)$, we use a Gaussian elimination, which expresses $A$ as the product of an invertible matrix $S(M \times M)$ and of a matrix $T(M \times N)$ in row-echelon form: the number of non-zero rows of $T$ is equal to the rank of $A$. 